\title{
Modeling of monitoring processes of structurally heterogeneous technological objects
}

\author{
Aleksandr Skatkov ${ }^{1}$, Victoria Shevchenko ${ }^{1}$, Dmitry Voronin ${ }^{1, *}$, and Dmitriy Moiseev ${ }^{2}$ \\ ${ }^{1}$ Sevastopol State University, IT\&CS department, 299053 Sevastopol, Russian Federation \\ ${ }^{2}$ ChVVMU, Sevastopol, Russian Federation
}

\begin{abstract}
The tasks of technological information capture, taken from industrial enterprise monitoring systems, and its further transfer to the level of management and decision-making are considered in the article. The problems arising at the stage of integration of isolated automation systems into a single information space of the enterprise have been analyzed. A model of the monitoring system of structurally heterogeneous technological objects has been proposed. It allows to estimate events' probabilities of the following types: successful information processing, computing equipment downtime.
\end{abstract}

\section{Introduction}

The modern automated control system of an industrial enterprise, as a rule, has an open architecture, including the following levels: object level (Field Management); level of data capture and dispatching (Process Management); enterprise resource management application level (Business Management) [1, 2].

The lower level (object level) contains different technological devices - sensors, controllers, electric drives, actuators - that are used for the implementation of regulatory and management actions on the primary controlled objects. Information flows got from lowerlevel systems should be available for all top-level applications and users. They can be used, for example, for analysis and control operations. The next level of management comprise SCADA (Supervisory Control And Data Acquisition) and DCS (Distributed Control Systems) systems. This level provides the following processes: 1) secondary processing of data obtained from primary monitoring systems; 2) organization of information storage and management of technological processes in production.

The production management systems are situated at the third level of the hierarchy. The main functions of this level are the following:

1) automated control systems integration;

2) creation of a common information space for production and technical data;

3) real-time processing and presentation of data got from the lower levels of automation. There are the following classes of information-control systems: MRP (Material Requirements Planning), MRP II (Production Resource Planning), ERP (Enterprise Resource Planning) и ERP II (Resource Management and Enterprise Relationships) [3, 4].
The actual task of industrial enterprises automation contains a single information space creation that will be able to perform the following functions:

- objective and rapid assessment of the current situation in the manufacture process;

- rapid optimal decision-making in enterprise management;

- elimination of information and organizational barriers between management and technological levels.

Technical, technological and organizational problems that arise at the stage of integration of heterogeneous systems, which control technological objects, are considered in this paper. A four-level model of data processing has been proposed. It allows to estimate the information processing quality for the entire CAM lifecycle, which starts from information capture (got from sensors and monitoring devices) and ends with distributed reporting information storage.

\section{Features analysis of the of automated enterprise systems integration into a single information space}

Integration of the existing enterprise automated systems into a single information and management economic infrastructure usually is carried out in the two directions: «horizontal» and «vertical».

The goal of horizontal integration is to ensure the real-time capture of data got from all systems that carry out control and management functions of technological process. They are the following: heterogeneous and territorially distributed automated process control systems (APCS), telemechanic systems, automated workplaces of manual information input, etc. The complexity of horizontal integration lies in the territorial

\footnotetext{
* Corresponding author: dima@,voronins.com
} 
distribution of existing APCS, variety of their data formats and software-technological tools.

Vertical integration provides automation of data exchange between the level of APCS and enterprise management level. To realize vertical integration, it's necessary to implement a reliable and efficient system providing processing and transmitting of data flows between APCS and automated enterprise management complexes. The following aspects should be mentioned: 1) APCS process data in real time; 2) automated enterprise management complexes use aggregated (synthetic) information for a certain period.

Analysis of modern information management systems architectures [5] allows to single out a generalized information exchange scheme that takes into account the horizontal and vertical integration of automated systems (fig. 1).

Thus, the data sources can be divided into three types: technological, laboratory and management. Technological are got from SCADA, DCS, PLC (programmable logic controller); laboratory were received from LIMS (Laboratory Information Management System), management - from the control room workstations. The main components of the information exchange scheme are data processing systems (DPS), which in turn are determined by a combination of program modules, data sets and technical means that ensure their storage and processing. Data is transmitted to real-time databases and DPS using specialized interfaces. Then it is accumulated in the specialized archives of long-term storage. The calculation of technological, technical and economic indicators is performed based on the collected input information and values from archival sources in DPS.

The storage duration of archives has two terms: short and long. By the degree of data retrieval the system files can be divided into the following types:

1) Realtime databases archives, which store information about the parameters of the production system obtained from monitoring;

2) Production time databases, which store information for technological purposes;

3) Databases and datamart designed to provide rapid information transfer to external systems.

Data archives also contain normative and reference information, supporting the decision-making process of operational dispatch and staff control. For these purposes the system includes specialized knowledge bases, analytical and simulation models complexes. To support the system structure management a complex of engineering administration is used. Special client applications allow to view the archived data using the required views or forms (graphics, tables, etc.). Monitoring systems used for distributed technological objects, as a rule, are not allocated to a separately functioning information system. Thus, they are realized

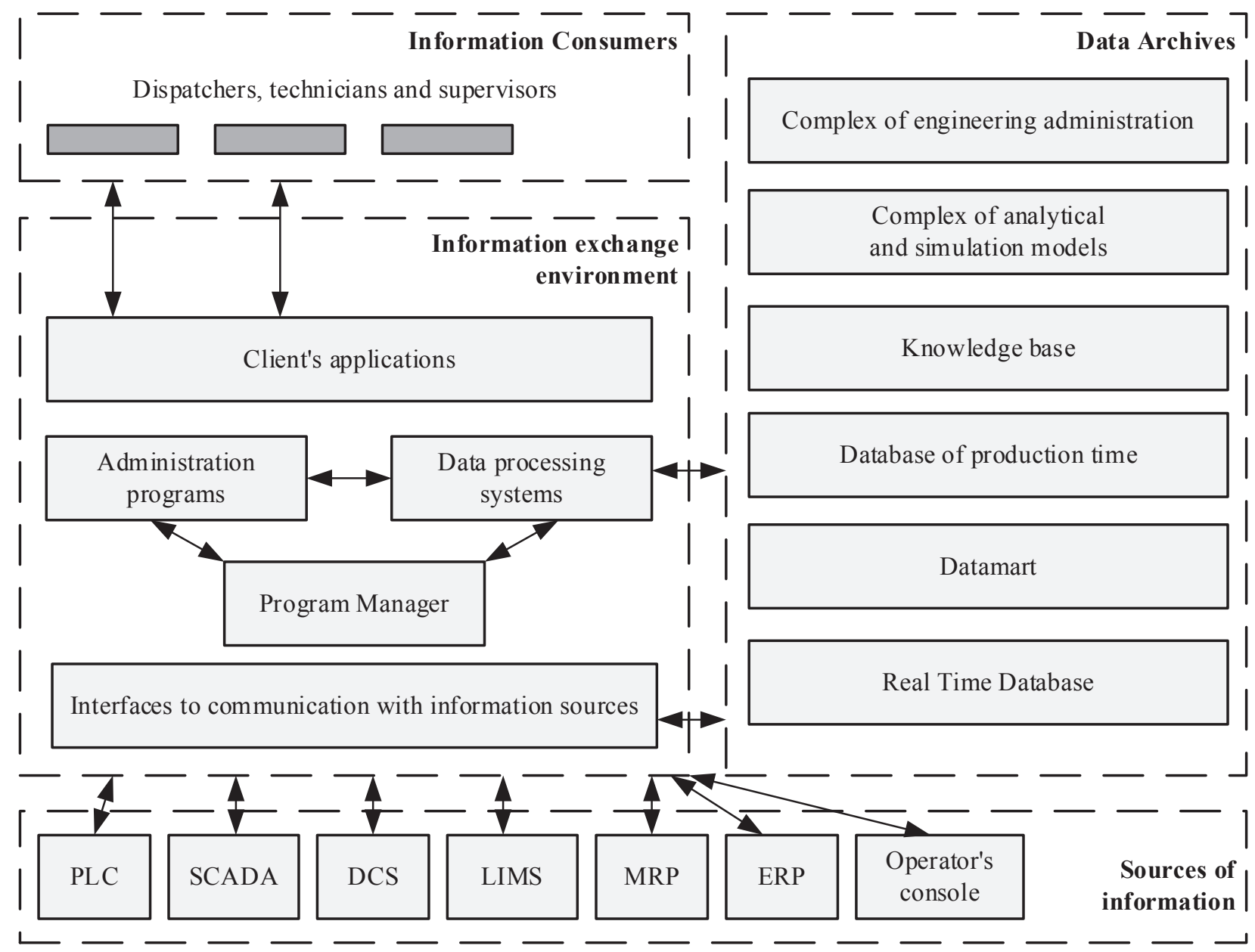

Fig.1. The scheme of information exchange in automated enterprise systems 


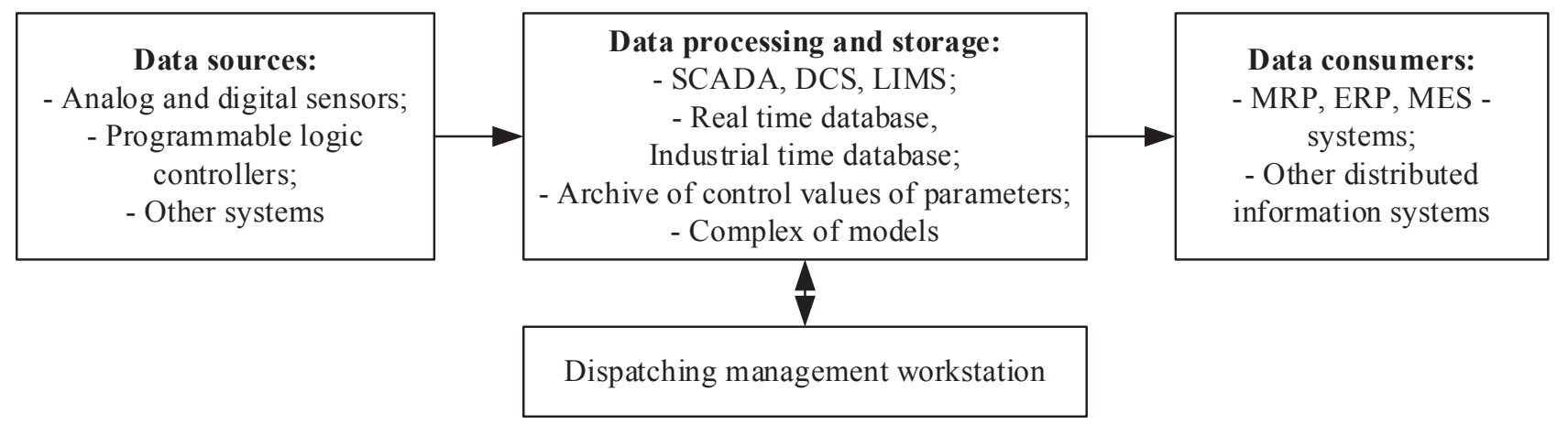

Fig.2. Information exchange scheme for monitoring systems

as a network of interacting information subsystems. This is due to the complexity of the structural and functional organization of the control object, large volume of controlled and processed parameters, territorial distribution, etc. Information exchange scheme for monitoring systems is represented on the fig. 2 . Nontriviality of the problem of vertical integration based on data got from monitoring information systems, APCS and CAM - is caused by the following factors:

- There is a large amount of primary data, and, as a result, there are problems with productive access to them by communication channels and storage systems;

- Complexity of integration processes administration and so on;

- $\quad$ Redundancy of primary data;

- The need to support a large number of industrial protocols for data access;

- Various manufacturers of APCS;

- The need to ensure information security in the exchange of data at the interface between the office and technological networks.

It should be noted that vertical unidirectional integration is required in the overwhelming majority of cases where monitoring systems are the sources and external information systems are the receivers of data.

\section{Conceptual model of the monitoring system for structurally-heterogeneous technological objects}

The main subsystems of distributed monitoring system are the following: subsystem of collection, accumulation, storage, retrieval of information dissemination [6] and data analysis subsystem [7]. Cloud computing technologies are used in modern industrial automation systems in order to save computing resources and to improve the management of information exchange and integration. A generalized scheme of the monitoring system is shown in fig. 3, a detailed description of data processing and storage networks based on cloud technologies is given in [8].

Regardless of the configuration of the computing infrastructure, when performing labor-intensive functional tasks in the cloud computing environment, the process of collecting and processing data, as a rule, includes four main stages [9]:

1) Data capture and data transfer from primary monitoring systems for multidimensional objects (sensors).
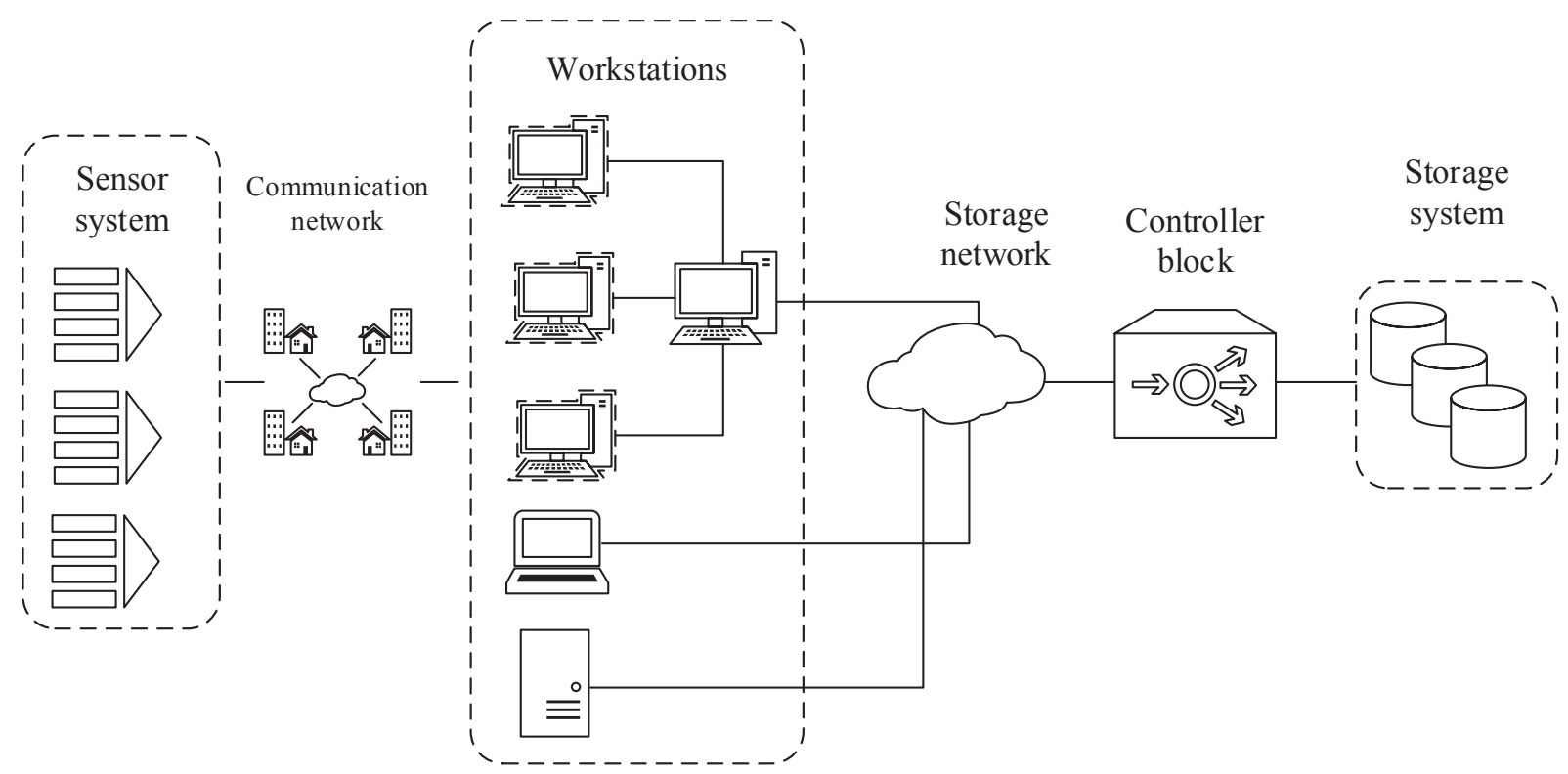

Fig.3. The scheme of the distributed monitoring system 
2) Primary data processing (filtering, classification and clustering processes).

3) Entering user data about the research process.

4) Archiving the information received during the working period.

Next, the formal model of the monitoring system for structurally-inhomogeneous objects is considered. It has been constructed using the approaches of the theory of queuing systems and graph representation of states and transitions of the system. The process of monitoring distributed production facilities can be represented by a four-phase queuing system with a finite number of states. The mentioned stages of data processing correspond to these phases (fig. 4).

In the first phase of processing, it is assumed that the number of sensors in the monitoring system is predetermined and data processing cycle - consistent, i.e. the information from the next sensor is processed only after processing of the previous data. In this case, the part of the computing infrastructure responsible for the current stage of data processing can be represented as a single-channel processing device.

The second phase of processing corresponds to the stage when the transmitted primary monitoring data is filtered and combined into clusters according to the classification characteristics indicated by users of the monitoring system (corresponds to the block of workstations in fig.3). At this stage, data belonging to the same cluster is combined into packets and is labeled for further processing. The model for the second phase of processing can be described using a multi-channel queuing system with limited queue. The issues of data clustering and description of the characteristic features of individual data packets lie outside the scope of the considered problem.

The third phase corresponds to the stage where data is consistently processed and supplemented by users. Status of data describe the class of its archival storage ("working array / online archive / long-term storage"). At this stage, the only channel of data processing is the workstation server.

The fourth phase corresponds to the stage of data archiving. To model the storage network, this phase can be represented as a multi-channel queuing system with a multidimensional incoming stream and a limited queue. A multidimensional input stream consists of three parts: the flow of writing data to a working array; operational archive; long-term storage archive.

The limitation of monitoring system consideration in conditions of stationary leads to serious systemic errors in decision-making. The reasons for the stationary disturbance can be the following: failures in the technological process of data processing, loss of information and retransmission of data in transmission networks and data storage networks, possible technical problems with the cloud infrastructure equipment or software, the human factor, etc. Therefore, more adequate is to present the monitoring process of a structurally heterogeneous object in the form of a multiphase non-stationary queuing system with variable values of the data entry and processing intensities. The development of the analytical model was based on the provisions works [10]. They have been extended taking into account the transition from a single-phase to a fourphase model and the specific features of distributed computing for production processes automation.

\section{Analytical model of the monitoring system}

The first processing phase is considered. It contains capturing and transfer operations with data got from the multidimensional object monitoring sensors. Consistently the system input receives $\mathrm{N}$ processing requests from the $\mathrm{N}$ system sensors. In order to simplify the analytical calculations let's suppose, that the distribution of time intervals between the moments of incoming requests are described by an exponential law with intensities $\left\{\lambda_{1}{ }^{(k)}, \ldots\right.$, $\left.\lambda_{i}{ }^{(k)}, \ldots, \lambda_{N}{ }^{(k)}\right\}$ depending on the request's number, where $k$ - is the processing phase number $(k=1,4), i-$ is the request's number. The law of processing times distribution is exponential (with intensities also depending on the request's number $-\left\{\mu_{I}^{(k)}, \ldots, \mu_{i}^{(k)}, \ldots, \mu_{N}^{(k)}\right\}$ respectively).

The state of the system at each time is characterized by the number of requests, denoted as $i=\{0, N\}$, and the number of processed requests, denoted as $j=\{0, N-i\}$. $P_{k, i, j}(t)$ denotes the probability of the event comprising the system's staying in the $(k, i, j)$-th state. The described system can be represented as a Markov chain with a discrete set of states and a continuous time.

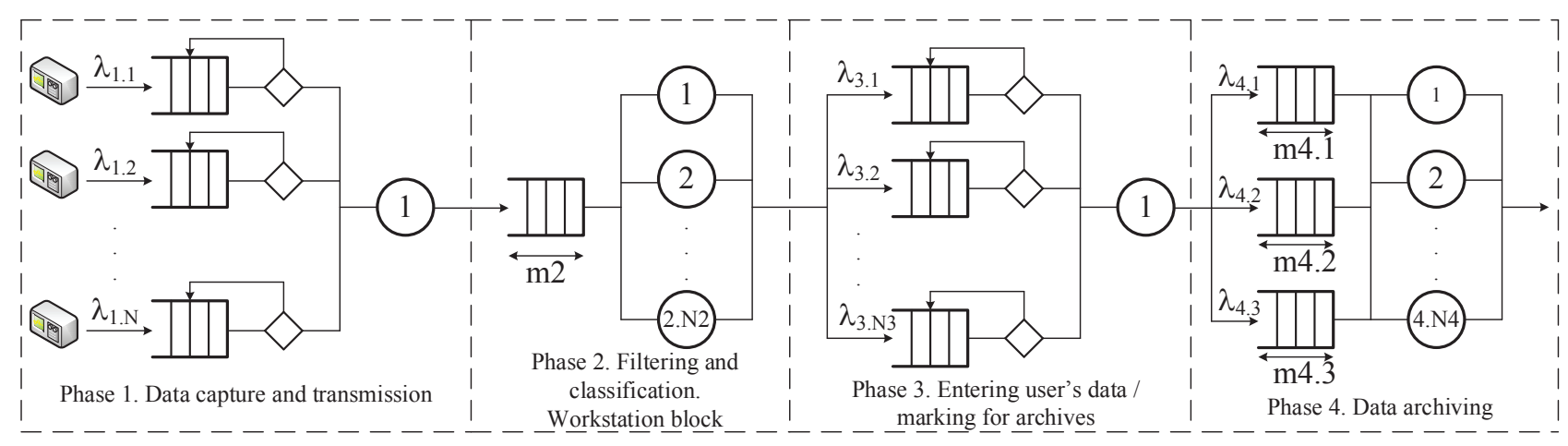

Fig.4. The scheme of the model of the data storage system used for objects monitoring 
The system status number is coded as: $<$ Processing phase number $>$ - is a number from 1 to 4 , corresponding to the number of the data processing phase in the distributed transmission and storage monitoring network; < Line number $>-$ is a number of unprocessed requests in the system; < Column number $>$ - is a number of requests that have been already processed in the system.

Transition from the state $(k, i, j)$ into the state $(k, i+1, j)$ indicates that the $(k, i+j+1)$-th request has entered the system. Transition from the $(k, i, j)$-th state into the $(k, i-1, j+1)$-th state indicates that the $(j+1)$-th request has been processed. For example, at the beginning of the simulation, the system is in the $(1,0,0)$ state. Then the system input receives a processing request - the transition to the $(1,1,0)$-th state accrues. Furthermore, if the system receives another request, it will come to the $(1,2,0)$-th state. If the single request have been successfully processed (data transfer to workstations in the primary processing system, see fig. 4) the system come in $(1,0,1)$-th state.

Total number of states $\mathrm{N}_{\mathrm{c}}$ is calculated by the formula (1) [11].

$$
N_{c}=\sum_{k=1}^{k=N+1} k
$$

A diagram of the transitions between system states for the first processing phase is shown on the fig.5.

To calculate the values of the probabilities of finding a system in each of the states, it is necessary to solve Kolmogorov's system of differential equations relatively to
$P_{k, i, j}(t)$, where $i, j$ - th equation in general form can be represented as (2).

$$
\begin{aligned}
\frac{d P_{k, i, j}}{d t} & =\delta(i)\left(P_{k, i-1, j} \lambda_{k, i+j}-P_{k, i, j} \mu_{k, j+1}\right)+ \\
& +\delta(j) P_{k, i+1, j-1} \mu_{k, j}- \\
& -\delta(N-i-j)\left(P_{k, i, j} \lambda_{k, i+j+1}\right)
\end{aligned}
$$

where $\delta(x)$ - Heaviside function.

To determine the values of the probabilities of finding the system in states $(k, i, j)$ the system of differential equations (2) should be decided on $P_{k, i, j}(t)$ under the following initial conditions: $P_{k, i, j}(0)=1$, if $i+j=0$ and $P_{k, i, j}(0)=0$ in other cases. The system of differential equations (2) can be solved in various ways, for example, using the Runge-Kutta method, which has an advantage of the simplicity of software implementation. The probability of $i$ requests being in the system at each point in time can be represented as (3).

$$
P_{k, i}(t)=\sum_{j=0}^{N-1} P_{k, i, j}(t)
$$

The (4)-th equation represents the expected number of requests in the system. The (5)-th equation describes the probability of $j$ requests proceeding at each point of time. The probability of proceeding of not less than $q$ requests at each point of time can be found using the (6)-th equation.

$$
N_{i}(t)=\sum_{i=1}^{N} i P_{k, i}(t)
$$

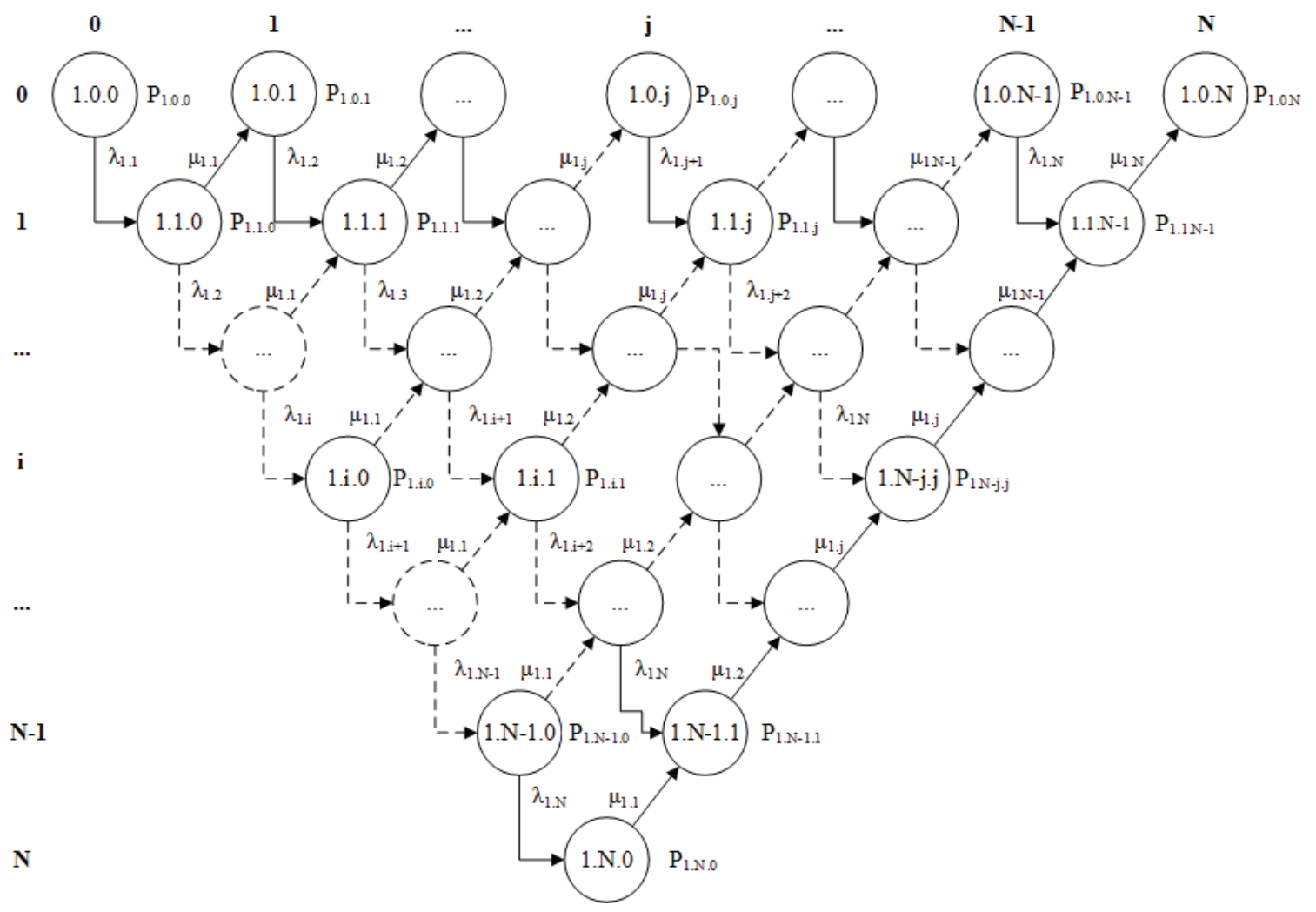

Fig. 5. Diagram of transitions between system states for the first phase of data processing in the monitoring system 


$$
\begin{gathered}
P_{k, j}(t)=\sum_{i=0}^{N-j} P_{k, i, j}(t) \\
P_{k, q}(t)=\sum_{i=q}^{N-j} P_{k, j}(t)
\end{gathered}
$$

Sources of non-stationarity of the system are the intensity of the arrival and proceeding time of requests, depending its number. An algorithm for calculating the probabilities of a similar type of model is given in works $[10,11]$.

The first data processing phase was considered to illustrate to usage of the proposed model. The following parameters were used: 1) arrival rates (requests per second): $\lambda_{1}=0.085$; $\lambda_{2}=0.08 ; \quad \lambda_{3}=0.075 ; \quad \lambda_{4}=0.07 ; \quad \lambda_{5}=0.065 ; \quad \lambda_{6}=0.06 ;$ $\left.\lambda_{7}=0.055 ; \lambda_{8}=0.05 ; \lambda_{9}=0.045 ; \lambda_{10}=0.04 ; 2\right)$ intensiveness of request processing: $\mu_{1}=0.18 ; \mu_{2}=0.14 ; \mu_{3}=0.1 ; \mu_{4}=0.6$; $\mu_{5}=0.2 ; \mu_{6}=0.1 ; \mu_{7}=0.08 ; \mu_{8}=0.07 ; \mu_{9}=0.06 ; \mu_{10}=0.05$.

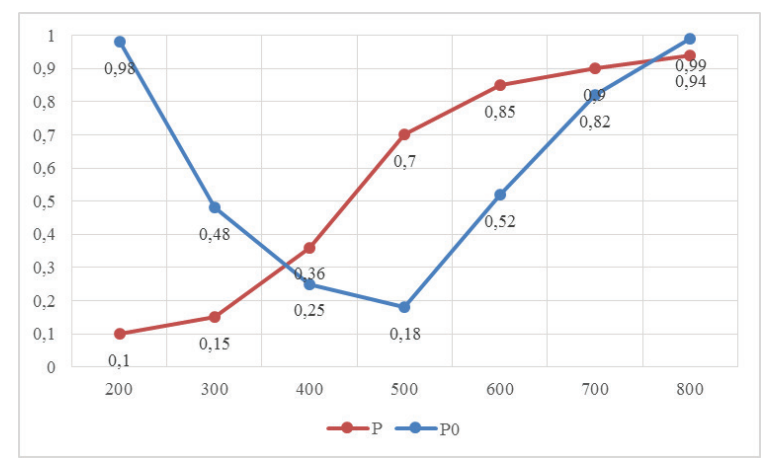

Fig. 6. The results of the calculation of the model of the first data processing phase in the monitoring system

On the fig. 6 the $\mathrm{P}$ curve shows the relationship between the probability of requests processing and the simulation time. Curve P0 shows dynamics of change in the probability of monitoring system's downtime.

\section{Conclusion}

The analytical model of the monitoring system, which has been proposed in this paper, can be used as an element of analytical and simulation models complex used in a decision-support system managing the integration of automated enterprise systems into a single information space. Its usage makes it possible to estimate such parameters as: probability of processing data streams by types; to determine the maximum package amount of requests that can be guaranteed processed in the system. These parameters are essential in assessing the quality of information processing for distributed heterogeneous monitoring objects.

Prospects for further research. Later on, based on the conceptual model of a four-phase queuing system, which has been proposed in this article, it is planned to develop a complex of simulation models for estimating the average processing time for requests packets by types, taking into account the dynamics of value and aging of monitoring information. It is also planned to conduct model experiments to fill the knowledge base of the decision support system for managing the integration of automated enterprise systems into a single information space.
This work was supported by the Russian Foundation for Basic Research (project № 15-29-07936).

\section{References}

1. A. A. Ambarcumjan, A. S. Hadeev, Analysis of the functionality of maintenance management systems and equipment repair, Problemy upravlenija 6, pp. 2-12, (2005).

2. A. V. Vicentij, E. V. Harionovskij, Technologies of data access in modern SCADA-systems, Trudy Kol'skogo nauchnogo centra RAN 7, pp. 203-210, (2011).

3. A.A Musaev, Integration of automated control systems for large industrial enterprises: principles, problems, solutions, Avtomatizacija v promyshlennosti 10, pp. 40-45, (2003).

4. Chyong Din Tjau, Interaction of open systems of industrial automation state and problems, Informacionno-upravljajushhie sistemy 2-3, pp. 5257, (2003).

5. D.P. Volodin, A.M. Marasanov, T.A. Tihonova, Architecture of distributed enterprise information system, Matematicheskoe modelirovanie 8, pp. 3136, (2002).

6. I.V. Hmel'nickaja, Methodological approaches to monitoring large-scale socio-economic systems, Jekonomika i upravlenie 1(33), pp. 100-103, (2012).

7. S.P. Kovaljov, Aspect-oriented approach to the design of monitoring systems for large-scale objects, Problemy informatiki 3(4), pp. 5-18, (2009).

8. Information Storage and Management: Storing, Managing, and Protecting Digital Information in Classic, Virtualized, and Cloud Environments (Piter, Saint-Petersburg, 2016) 544.

9. A. Skatkov, E. Mashchenko, V. Shevchenko, D. Voronin, Actors Interactions Research in Cloud Computing Environments Using System Dynamics Methodology, Proceedings of the 18th FRUCT \& ISPIT Conference, Saint-Petersburg, Russia. FRUCT Oy, Finland, pp - 612-619 (2016).

10. V.P. Bubnov, V.I. Safonov, Development of dynamic models of non-stationary service systems (Lan', Saint-Petersburg, 1999) 64.

11. V.P. Bubnov, A. S. Eremin, S. A. Sergeev. Features of software implementation of the numerical and analytical method for calculating models of nonstationary service systems, Trudy SPIIRAN 1(38), pp. 218-232, (2015). 